\title{
Post-Newtonian effects on the stability of the triangular solution in the three-body problem for general masses
}

\author{
Kei Yamada, ${ }^{1, *}$ Takuya Tsuchiya, ${ }^{2}$ and Hideki Asada ${ }^{3}$ \\ 1 Department of Physics, Kyoto University, Kyoto 606-8502, Japan \\ 2 Department of Mathematical Sciences, \\ Waseda University, Shinjuku 169-8555, Japan \\ 3 Faculty of Science and Technology, \\ Hirosaki University, Hirosaki 036-8561, Japan
}

\begin{abstract}
Continuing work initiated in earlier publications [Ichita, Yamada and Asada, Phys. Rev. D 83, 084026 (2011); Yamada and Asada, Phys. Rev. D 86, 124029 (2012)], we examine the postNewtonian $(\mathrm{PN})$ effects on the stability of the triangular solution in the relativistic three-body problem for general masses. For three finite masses, a condition for stability of the triangular solution is obtained at the first post-Newtonian (1PN) order, and it recovers previous results for the PN restricted three-body problem when one mass goes to zero. The stability regions still exist even at the $1 \mathrm{PN}$ order, though the $\mathrm{PN}$ triangular configuration for general masses is less stable than the PN restricted three-body case as well as the Newtonian one.
\end{abstract}

PACS numbers: 04.25.Nx, 45.50.Pk, 95.10.Ce, 95.30.Sf

*Electronic address: k.yamada@tap.scphys.kyoto-u.ac.jp 


\section{INTRODUCTION}

One of classical problems in astronomy and physics is the three-body problem in Newtonian gravity (e.g., [1 3] $]$ ). The gravitational three-body problem is not integrable by analytical methods. As particular solutions, however, Euler and Lagrange found a collinear solution and an equilateral triangular one, respectively. The solutions to the restricted three-body problem, where one of the three bodies is a test particle, are known as Lagrangian points $L_{1}, L_{2}, L_{3}, L_{4}$ and $L_{5}$ [1]. Lagrange's equilateral triangular solution has also a practical importance, since $L_{4}$ and $L_{5}$ for the Sun-Jupiter system are stable and indeed the Trojan asteroids are located there. Even for the Sun-Earth system, asteroids were also found around $L_{4}$ by recent observations [4].

Recently, Lagrangian points have attracted renewed interests for relativistic astrophysics 5 -10], where they have discussed the post-Newtonian (PN) corrections for Lagrangian points [5, 6], and the gravitational radiation reaction on $L_{4}$ and $L_{5}$ analytically [7] and by numerical methods [8-10]. It is currently important to reexamine Lagrangian points in the framework of general relativity. As a pioneering work [11], Nordtvedt has pointed out that the location of the triangular points is very sensitive to the ratio of the gravitational mass to the inertial one, though his analysis does not fully take account of the first post-Newtonian (1PN) terms. Along this course, it might be important as a gravity experiment to discuss the three-body coupling terms in the $\mathrm{PN}$ force, because some of the terms are proportional to a product of three masses such as $m_{1} \times m_{2} \times m_{3}$. Such a triple product appears only for relativistic three (or more) body systems but it does not for relativistic compact binaries nor Newtonian three-body systems. In addition, it has been pointed out that three-body interactions might play important roles for compact binary mergers in hierarchical triple systems [12 16]. Very recently, moreover, a first relativistic hierarchical triple system has been discovered by Ransom and his collaborators [17].

For three finite masses, in the 1PN approximation, the existence and uniqueness of a PN collinear solution corresponding to Euler's one have been shown by Yamada and Asada [18, 19]. Also, Ichita et al., including two of the present authors, have shown that an equilateral triangular solution is possible at the $1 \mathrm{PN}$ order, if and only if all the three masses are equal [20]. Generalizing this earlier work, Yamada and Asada have found a $P N$ triangular equilibrium solution for general masses with $1 \mathrm{PN}$ corrections to each side length 
[21]. This PN triangular configuration for general masses is not always equilateral and it recovers the previous results by Krefetz [5] and Maindl [6] for the restricted three-body case.

In Newtonian gravity, Gascheau proved that Lagrange's equilateral triangular configuration for circular motion is stable [22], if

$$
\frac{m_{1} m_{2}+m_{2} m_{3}+m_{3} m_{1}}{\left(m_{1}+m_{2}+m_{3}\right)^{2}}<\frac{1}{27} .
$$

Routh extended the result to a general law of gravitation $\propto 1 / r^{k}$, and found the condition for stability as [23]

$$
\frac{m_{1} m_{2}+m_{2} m_{3}+m_{3} m_{1}}{\left(m_{1}+m_{2}+m_{3}\right)^{2}}<\frac{1}{3}\left(\frac{3-k}{1+k}\right)^{2} .
$$

For the restricted three-body limit as $m_{3} \rightarrow 0$, Douskos and Perdios examined the stability of $L_{4}\left(L_{5}\right)$ in the 1PN approximation, and they obtained a region of stability as [24]

$$
\frac{m_{2}}{m_{1}+m_{2}}<\mu_{0}-\frac{17 \sqrt{69}}{486} \varepsilon
$$

where we assume $m_{1}>m_{2}$ without loss of generality, the Newtonian value $\mu_{0}=(9-\sqrt{69}) / 18$, and we define

$$
\varepsilon \equiv \frac{G M}{c^{2} \ell}
$$

for the total mass $M$ and each side length $\ell$ of the Newtonian equilateral triangle. Singh and Bello also discussed the stability in the restricted three-body problem by taking account of not only the general relativistic effects but also radiation pressure and a small perturbation in the centrifugal force [25, 26]. For three finite masses, however, it is not clear whether the PN triangular configuration is stable. In this paper, we study this issue by fully taking account of all the $1 \mathrm{PN}$ terms.

This paper is organized as follows. In Sec. II, we briefly summarize the PN triangular equilibrium solution for three finite masses. In Sec. III, we consider the stability of the solution at the 1PN order. Section IV is devoted to the conclusion. Hereafter, we take the units of $G=c=1$.

\section{A POST-NEWTONIAN TRIANGULAR SOLUTION}

In this section, following Ref. [21], we summarize a derivation of the PN triangular solution to the relativistic three-body problem for general masses. We take account of the 
terms at the 1PN order by employing the Einstein-Infeld-Hoffman (EIH) equations of motion in the standard PN coordinates as [27-30]

$$
\begin{aligned}
m_{K} \frac{d^{2} \boldsymbol{r}_{K}}{d t^{2}}= & \sum_{A \neq K} \boldsymbol{r}_{A K} \frac{m_{K} m_{A}}{r_{A K}^{3}}\left[1-4 \sum_{B \neq K} \frac{m_{B}}{r_{B K}}-\sum_{C \neq A} \frac{m_{C}}{r_{C A}}\left(1-\frac{\boldsymbol{r}_{A K} \cdot \boldsymbol{r}_{C A}}{2 r_{C A}^{2}}\right)\right. \\
& \left.+v_{K}^{2}+2 v_{A}^{2}-4 \boldsymbol{v}_{A} \cdot \boldsymbol{v}_{K}-\frac{3}{2}\left(\boldsymbol{v}_{A} \cdot \boldsymbol{n}_{A K}\right)^{2}\right] \\
& -\sum_{A \neq K}\left(\boldsymbol{v}_{A}-\boldsymbol{v}_{K}\right) \frac{m_{K} m_{A}}{r_{A K}^{2}} \boldsymbol{n}_{A K} \cdot\left(3 \boldsymbol{v}_{A}-4 \boldsymbol{v}_{K}\right)+\frac{7}{2} \sum_{A \neq K} \sum_{C \neq A} \boldsymbol{r}_{C A} \frac{m_{K} m_{A} m_{C}}{r_{A K} r_{C A}^{3}},
\end{aligned}
$$

where $\boldsymbol{r}_{I}$ and $\boldsymbol{v}_{I}$ denote the location and the velocity of each body in an inertial frame and we define

$$
\begin{aligned}
\boldsymbol{r}_{I J} & \equiv \boldsymbol{r}_{I}-\boldsymbol{r}_{J}, \\
r_{I J} & \equiv\left|\boldsymbol{r}_{I J}\right|, \\
\boldsymbol{n}_{I J} & \equiv \frac{\boldsymbol{r}_{I J}}{r_{I J}}
\end{aligned}
$$

In the following, we assume circular motion.

We consider a PN triangular configuration with $1 \mathrm{PN}$ corrections to each side length of a Newtonian equilateral triangle, so that the distances between the bodies are

$$
r_{I J}=\ell\left(1+\rho_{I J}\right)
$$

where $I, J=1,2,3$ and $\rho_{I J}\left(=\rho_{J I}\right)$ is dimensionless PN corrections (see Fig. 1). Because of the circular motion, $\ell$ and $\rho_{I J}$ are constants. Note that we neglect the terms of second (and higher) order in $\varepsilon$ henceforth. Here, if all the three corrections are equal (i.e. $\rho_{12}=$ $\rho_{23}=\rho_{31}=\rho$ ), a PN configuration is still an equilateral triangle, though each side length is changed by a scale transformation as $\ell \rightarrow \ell(1+\rho)$. Namely, one of the degrees of freedom for the PN corrections corresponds to a scale transformation, and this is unimportant. In order to eliminate this degree of freedom, we impose a constraint condition

$$
\frac{r_{12}+r_{23}+r_{31}}{3}=\ell
$$

which means that the arithmetical mean of the three distances of the bodies is not changed by the PN corrections. Namely,

$$
\rho_{12}+\rho_{23}+\rho_{31}=0
$$


Please see also Ref. [21] for imposing this constraint.

Let $\omega_{I}$ denote the angular velocities of the Ith body with PN corrections. The EIH equation of motion for $m_{1}$ becomes

$$
\begin{aligned}
-\omega_{1}^{2} \boldsymbol{r}_{\mathrm{N} 1}= & -\frac{M}{\ell^{3}} \boldsymbol{r}_{\mathrm{N} 1}+\boldsymbol{\delta}_{\mathrm{EIH} 1} \varepsilon-\frac{3}{2} \frac{M}{\ell^{2}} \frac{1}{\sqrt{\nu_{2}^{2}+\nu_{2} \nu_{3}+\nu_{3}^{2}}} \\
& \times\left\{\left[\nu_{2}\left(\nu_{1}-\nu_{2}-1\right) \rho_{12}+\nu_{3}\left(\nu_{1}-\nu_{3}-1\right) \rho_{31}\right] \boldsymbol{n}_{1}+\sqrt{3} \nu_{2} \nu_{3}\left(\rho_{12}-\rho_{31}\right) \boldsymbol{n}_{\perp 1}\right\},
\end{aligned}
$$

where the mass ratio $\nu_{I} \equiv m_{I} / M, \boldsymbol{r}_{\mathrm{N} I}$ and $\boldsymbol{v}_{\mathrm{N} I}$ are the Newtonian location and velocity, respectively, $\boldsymbol{n}_{I} \equiv \boldsymbol{r}_{\mathrm{N} I} / r_{\mathrm{N} I}$, and $\boldsymbol{n}_{\perp I} \equiv \boldsymbol{v}_{\mathrm{N} I} / v_{\mathrm{N} I}$. Note that $\boldsymbol{n}_{I} \perp \boldsymbol{n}_{\perp I}$ in the circular motion. The PN term $\boldsymbol{\delta}_{\mathrm{EIH} 1}$ is defined by

$$
\begin{aligned}
\boldsymbol{\delta}_{\mathrm{EIH} 1}= & \frac{1}{16} \frac{M}{\ell^{2}} \frac{1}{\sqrt{\nu_{2}^{2}+\nu_{2} \nu_{3}+\nu_{3}^{2}}} \\
& \times\left\{\left\{16\left(\nu_{2}^{2}+\nu_{2} \nu_{3}+\nu_{3}^{2}\right)\left[3-\left(\nu_{1} \nu_{2}+\nu_{2} \nu_{3}+\nu_{3} \nu_{1}\right)\right]\right.\right. \\
& \left.+9 \nu_{2} \nu_{3}\left[2\left(\nu_{2}+\nu_{3}\right)+\nu_{2}^{2}+4 \nu_{2} \nu_{3}+\nu_{3}^{2}\right]\right\} \boldsymbol{n}_{1} \\
& \left.+3 \sqrt{3} \nu_{2} \nu_{3}\left(\nu_{2}-\nu_{3}\right)\left(5-3 \nu_{1}\right) \boldsymbol{n}_{\perp 1}\right\} .
\end{aligned}
$$

One can obtain the equations of motion for $m_{2}$ and $m_{3}$ by cyclic manipulations as $1 \rightarrow 2 \rightarrow$ $3 \rightarrow 1$

The PN triangular configuration becomes an equilibrium solution in the circular motion if and only if the following conditions (a) and (b) simultaneously hold: (a) the term proportional to $\boldsymbol{n}_{\perp I}$ vanishes and (b) all the angular velocities are the same.

As a result, we obtain the PN corrections that satisfy the above conditions as [21]

$$
\begin{aligned}
& \rho_{12}=\frac{1}{24}\left[\left(\nu_{2}-\nu_{3}\right)\left(5-3 \nu_{1}\right)-\left(\nu_{3}-\nu_{1}\right)\left(5-3 \nu_{2}\right)\right] \varepsilon \\
& \rho_{23}=\frac{1}{24}\left[\left(\nu_{3}-\nu_{1}\right)\left(5-3 \nu_{2}\right)-\left(\nu_{1}-\nu_{2}\right)\left(5-3 \nu_{3}\right)\right] \varepsilon \\
& \rho_{31}=\frac{1}{24}\left[\left(\nu_{1}-\nu_{2}\right)\left(5-3 \nu_{3}\right)-\left(\nu_{2}-\nu_{3}\right)\left(5-3 \nu_{1}\right)\right] \varepsilon
\end{aligned}
$$

which give a PN triangular equilibrium solution for general masses. In this case, the angular velocities of the bodies are equal (i.e. $\left.\omega_{1}=\omega_{2}=\omega_{3}=\omega\right)$. These corrections for the restricted three-body problem as $\nu_{3} \rightarrow 0$ reduce to previous results [5, 6].

Substituting Eq. (14) and Eq. (16) into Eq. (12), we obtain the angular velocity of the three bodies

$$
\omega=\omega_{\mathrm{N}}\left(1+\tilde{\omega}_{\mathrm{PN}}\right)
$$


where the Newtonian angular velocity $\omega_{\mathrm{N}}=\sqrt{M / \ell^{3}}$ and the dimensionless 1PN correction

$$
\tilde{\omega}_{\mathrm{PN}}=-\frac{1}{16}\left[29-14\left(\nu_{1} \nu_{2}+\nu_{2} \nu_{3}+\nu_{3} \nu_{1}\right)\right] \varepsilon .
$$

Using a relation $\nu_{1}+\nu_{2}+\nu_{3}=1$, we can show $\tilde{\omega}_{\mathrm{PN}}<0$, that is, $\omega<\omega_{\mathrm{N}}$ for the fixed system parameters $\ell$ and $M$. In other words, the PN triangular configuration is always smaller than the Newtonian one if the masses and angular velocity of the three bodies are fixed.

\section{THE STABILITY OF THE POST-NEWTONIAN TRIANGULAR SOLUTION}

Next, we study the stability of the PN triangular solution by taking account of linear perturbations in the orbital plane. It is convenient to use the corotating coordinates with the origin as the center of mass even after adding perturbations. Therefore, the number of the degrees of freedom for the perturbations decreases from six to four. One of them corresponds to a perturbation in the angular velocity, and the three other perturbations denote changes in the shape and size of the PN triangle.

We consider two perturbations corresponding to changes in the distances $r_{12}$ and $r_{31}$ as

$$
\begin{aligned}
& r_{12}=\ell\left(1+\rho_{12}+\delta \chi_{12}\right), \\
& r_{31}=\ell\left(1+\rho_{31}+\delta \chi_{31}\right),
\end{aligned}
$$

where $\chi_{12}$ and $\chi_{31}$ are perturbations in the distances and $\delta$ is a bookkeeping parameter that denotes the smallness of the perturbations. And, a perturbation $\psi_{23}$ denotes a change in the angle $\varphi_{23}$ between $r_{12}$ and $r_{31}$ as

$$
\varphi_{23}=\frac{\pi}{3}+\sqrt{3} \rho_{23}+\delta \psi_{23} .
$$

These three perturbations mean changes in the shape and size of the PN triangle. For the remaining one of the degrees of freedom corresponding to a change in the angular velocity, we denote

$$
\theta_{12}=\Theta_{12}+\delta \sigma
$$

where $\theta_{12}$ and $\sigma$ denote the direction of $\boldsymbol{r}_{12}$ to the reference frame and a perturbation in it, respectively. $\Theta_{12}$ is the unperturbed direction, which satisfies the equation as

$$
\frac{d \Theta_{12}}{d t}=\omega_{\mathrm{N}}\left(1+\tilde{\omega}_{\mathrm{PN}}\right)
$$


Note that the $1 \mathrm{PN}$ corrections $\rho_{I J}$ satisfy Eq. (11), while the perturbations are arbitrary. Figure 2 shows a schematic figure for these four perturbations. The above choice of the perturbations is convenient, because we can avoid directly using the PN center of mass [27-29].

Note that the perturbations $\chi_{12}, \chi_{31}, \psi_{23}$, and $\sigma$ have not only the Newtonian terms but also the $1 \mathrm{PN}$ ones. For instance, the perturbation $\sigma$ can be expanded as

$$
\sigma=\sigma_{\mathrm{N}}+\sigma_{\mathrm{PN}}
$$

where $\sigma_{\mathrm{N}}$ and $\sigma_{\mathrm{PN}}(=\mathrm{O}(\varepsilon))$ are the Newtonian term and the $1 \mathrm{PN}$ one, respectively. In the following, we neglect the terms of second (and higher) order in $\delta$. Namely, we calculate to the terms of order $\varepsilon \times \delta$ (i.e. the linear perturbation at the $1 \mathrm{PN}$ order).

Defining a new variable as $X \equiv \chi_{31}-\chi_{12}$, we obtain the equation of motion for $\boldsymbol{r}_{12}$ (see Appendix A for a detailed derivation of the equations). Its radial part is

$$
\begin{aligned}
& {\left[\left(D^{2}-3\right) \chi_{12}-2 D \sigma-\frac{9}{4} \nu_{3} X-\frac{3 \sqrt{3}}{4} \nu_{3} \psi_{23}\right]+\varepsilon\left[-\frac{1}{32}\left\{4 \sqrt{3}\left(\nu_{1}-\nu_{2}\right)\left(7-9 \nu_{3}\right) \nu_{3} D\right.\right.} \\
& +\left(36 \nu_{2}^{3}+234 \nu_{1} \nu_{2}^{2}-146 \nu_{2}^{2}+261 \nu_{1}^{2} \nu_{2}-488 \nu_{1} \nu_{2}+155 \nu_{2}+63 \nu_{1}^{3}-155 \nu_{1}^{2}+137 \nu_{1}\right. \\
& -585)\} \chi_{12}-\frac{1}{24}\left(27 \nu_{2}^{3}+135 \nu_{1} \nu_{2}^{2}-21 \nu_{2}^{2}+135 \nu_{1}^{2} \nu_{2}-210 \nu_{1} \nu_{2}+24 \nu_{2}+27 \nu_{1}^{3}-21 \nu_{1}^{2}\right. \\
& \left.+24 \nu_{1}-155\right) D \sigma-\frac{1}{32} \nu_{3}\left\{4 \sqrt{3}\left(9 \nu_{1} \nu_{2}+10 \nu_{2}+9 \nu_{1}^{2}-6 \nu_{1}-4\right) D-\left(216 \nu_{2}^{2}+288 \nu_{1} \nu_{2}\right.\right. \\
& \left.\left.-154 \nu_{2}+171 \nu_{1}^{2}-38 \nu_{1}+420\right)\right\} X+\frac{1}{32} \nu_{3}\left\{4 \left(18 \nu_{2}^{2}+27 \nu_{1} \nu_{2}-2 \nu_{2}+9 \nu_{1}^{2}+14 \nu_{1}\right.\right. \\
& \left.\left.-12) D+\sqrt{3}\left(51 \nu_{2}^{2}+114 \nu_{1} \nu_{2}+2 \nu_{2}+87 \nu_{1}^{2}-120 \nu_{1}+155\right)\right\} \psi_{23}\right]=0,
\end{aligned}
$$


and the tangential part is

$$
\begin{aligned}
& {\left[2 D \chi_{12}+D^{2} \sigma-\frac{3 \sqrt{3}}{4} \nu_{3} X+\frac{9}{4} \nu_{3} \psi_{23}\right]+\varepsilon\left[-\frac{1}{32}\left\{4 \left(9 \nu_{2}^{3}+45 \nu_{1} \nu_{2}^{2}+9 \nu_{2}^{2}+45 \nu_{1}^{2} \nu_{2}\right.\right.\right.} \\
& \left.-30 \nu_{1} \nu_{2}-18 \nu_{2}+9 \nu_{1}^{3}+9 \nu_{1}^{2}-18 \nu_{1}+61\right) D+3 \sqrt{3} \nu_{3}\left(12 \nu_{2}^{2}-6 \nu_{1} \nu_{2}+14 \nu_{2}-15 \nu_{1}^{2}\right. \\
& \left.\left.+4 \nu_{1}-5\right)\right\} \chi_{12}-\frac{1}{24}\left\{\left(3 \nu_{2}^{2}+12 \nu_{1} \nu_{2}-18 \nu_{2}+3 \nu_{1}^{2}-18 \nu_{1}+10\right) D^{2}-3 \sqrt{3}\left(\nu_{1}-\nu_{2}\right)\right. \\
& \left.\times \nu_{3}\left(9 \nu_{2}+9 \nu_{1}+4\right) D\right\} \sigma+\frac{1}{32} \nu_{3}\left\{4\left(18 \nu_{2}^{2}+27 \nu_{1} \nu_{2}+8 \nu_{2}+9 \nu_{1}^{2}+16 \nu_{1}-12\right) D\right. \\
& \left.+\sqrt{3}\left(36 \nu_{2}^{2}+72 \nu_{1} \nu_{2}-54 \nu_{2}+81 \nu_{1}^{2}-90 \nu_{1}+160\right)\right\} X+\frac{1}{32} \nu_{3}\left\{4 \sqrt { 3 } \left(9 \nu_{1} \nu_{2}+8 \nu_{2}\right.\right. \\
& \left.\left.\left.+9 \nu_{1}^{2}-4\right) D-9\left(21 \nu_{2}^{2}+14 \nu_{1} \nu_{2}-10 \nu_{2}+13 \nu_{1}^{2}-8 \nu_{1}+45\right)\right\} \psi_{23}\right]=0,
\end{aligned}
$$

where $D$ denotes a differential operator with respect to a normalized time $\tilde{t} \equiv \omega_{\mathrm{N}} t$.

In the same way, we obtain the equation of motion for $\boldsymbol{r}_{31}$ and its radial and tangential parts are

$$
\begin{aligned}
& {\left[\left(D^{2}-3\right) \chi_{12}-2 D \sigma+\left(D^{2}-3+\frac{9}{4} \nu_{2}\right) X-\left(2 D+\frac{3 \sqrt{3}}{4} \nu_{2}\right) \psi_{23}\right]+\varepsilon\left[-\frac{1}{32}\{4 \sqrt{3}\right.} \\
& \times\left(\nu_{3}-\nu_{1}\right)\left(7-9 \nu_{2}\right) \nu_{2} D+\left(36 \nu_{3}^{3}+234 \nu_{1} \nu_{3}^{2}-146 \nu_{3}^{2}+261 \nu_{1}^{2} \nu_{3}-488 \nu_{1} \nu_{3}+155 \nu_{3}\right. \\
& \left.\left.+63 \nu_{1}^{3}-155 \nu_{1}^{2}+137 \nu_{1}-585\right)\right\} \chi_{12}-\frac{1}{24}\left(27 \nu_{3}^{3}+135 \nu_{1} \nu_{3}^{2}-21 \nu_{3}^{2}+135 \nu_{1}^{2} \nu_{3}-210 \nu_{1} \nu_{3}\right. \\
& \left.+24 \nu_{3}+27 \nu_{1}^{3}-21 \nu_{1}^{2}+24 \nu_{1}-155\right) D \sigma-\frac{1}{32}\left\{4 \sqrt{3} \nu_{2}\left(9 \nu_{3}^{2}+9 \nu_{1} \nu_{3}+8 \nu_{3}-4 \nu_{1}-4\right) D\right. \\
& -\left(180 \nu_{3}^{3}+270 \nu_{1} \nu_{3}^{2}-224 \nu_{3}^{2}+198 \nu_{1}^{2} \nu_{3}+8 \nu_{1} \nu_{3}+419 \nu_{3}+108 \nu_{1}^{3}-54 \nu_{1}^{2}+321 \nu_{1}\right. \\
& +165)\} X+\frac{1}{96}\left\{4\left(27 \nu_{3}^{3}-39 \nu_{3}^{2}-27 \nu_{1}^{2} \nu_{3}+165 \nu_{1} \nu_{3}-54 \nu_{3}+36 \nu_{1}^{2}-102 \nu_{1}+191\right) D\right. \\
& \left.\left.+3 \sqrt{3} \nu_{2}\left(51 \nu_{3}^{2}+114 \nu_{1} \nu_{3}+2 \nu_{3}+87 \nu_{1}^{2}-120 \nu_{1}+155\right)\right\} \psi_{23}\right]=0
\end{aligned}
$$




$$
\begin{aligned}
& {\left[2 D \chi_{12}+D^{2} \sigma+\left(2 D-\frac{3 \sqrt{3}}{4} \nu_{2}\right) X+\left(D^{2}-\frac{9}{4} \nu_{2}\right) \psi_{23}\right]+\varepsilon\left[-\frac{1}{32}\left\{4 \left(9 \nu_{3}^{3}+45 \nu_{1} \nu_{3}^{2}\right.\right.\right.} \\
& \left.+9 \nu_{3}^{2}+45 \nu_{1}^{2} \nu_{3}-30 \nu_{1} \nu_{3}-18 \nu_{3}+9 \nu_{1}^{3}+9 \nu_{1}^{2}-18 \nu_{1}+61\right) D-3 \sqrt{3} \nu_{2}\left(12 \nu_{3}^{2}-6 \nu_{1} \nu_{3}\right. \\
& \left.\left.+14 \nu_{3}-15 \nu_{1}^{2}+4 \nu_{1}-5\right)\right\} \chi_{12}-\frac{1}{24}\left\{\left(3 \nu_{3}^{2}+12 \nu_{1} \nu_{3}-18 \nu_{3}+3 \nu_{1}^{2}-18 \nu_{1}+10\right) D^{2}\right. \\
& \left.-3 \sqrt{3}\left(\nu_{3}-\nu_{1}\right)\left(13-9 \nu_{2}\right) \nu_{2} D\right\} \sigma+\frac{1}{32}\left\{4 \left(9 \nu_{3}^{3}-19 \nu_{3}^{2}-9 \nu_{1}^{2} \nu_{3}+27 \nu_{1} \nu_{3}-2 \nu_{3}-2 \nu_{1}^{2}\right.\right. \\
& \left.\left.-10 \nu_{1}-49\right) D+\sqrt{3}\left(72 \nu_{3}^{2}+54 \nu_{1} \nu_{3}-12 \nu_{3}+36 \nu_{1}^{2}-78 \nu_{1}+145\right) \nu_{2}\right\} X-\frac{1}{96}\left\{4 \left(3 \nu_{3}^{2}\right.\right. \\
& \left.+12 \nu_{1} \nu_{3}-18 \nu_{3}+3 \nu_{1}^{2}-18 \nu_{1}+10\right) D^{2}-12 \sqrt{3}\left(9 \nu_{3}^{2}+9 \nu_{1} \nu_{3}+12 \nu_{3}-4 \nu_{1}-4\right) \nu_{2} D \\
& \left.\left.-27\left(21 \nu_{3}^{2}+14 \nu_{1} \nu_{3}-10 \nu_{3}+13 \nu_{1}^{2}-8 \nu_{1}+45\right) \nu_{2}\right\} \psi_{23}\right]=0 .
\end{aligned}
$$

First, we study the condition for stability in Newtonian gravity. In the Newtonian limit $\varepsilon \rightarrow 0$, the equations of motion (25) - (28) for the perturbations are rearranged as

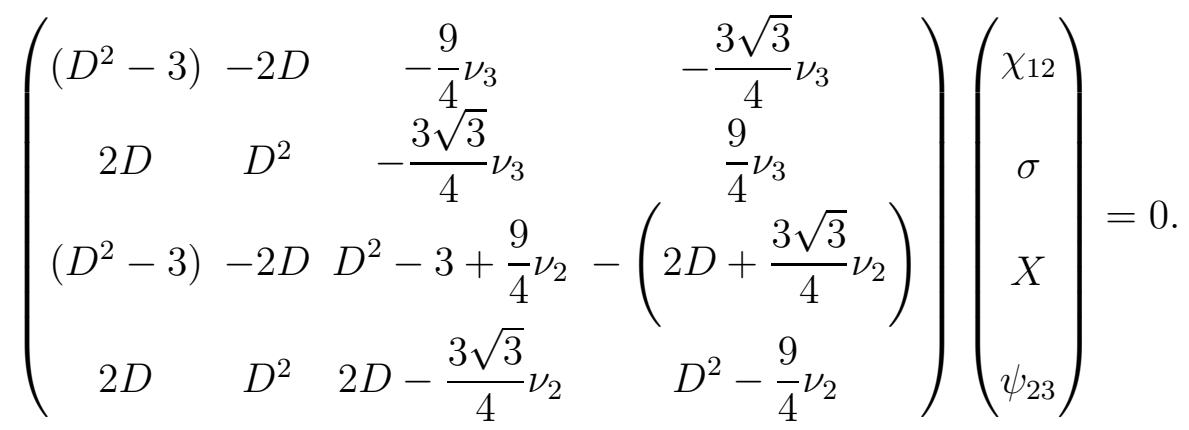

Carrying out a classical stability analysis (e.g. [2]), we obtain the eigenvalue equation, so called the secular equation as

$$
\lambda^{2}\left(\lambda^{2}+1\right)\left(\lambda^{2}+\frac{1+\sqrt{1-27 V}}{2}\right)\left(\lambda^{2}+\frac{1-\sqrt{1-27 V}}{2}\right)=0,
$$

where $\lambda$ is the eigenvalue and $V \equiv \nu_{1} \nu_{2}+\nu_{2} \nu_{3}+\nu_{3} \nu_{1}$. Since Eq. (30) has only the terms of the even-order of $\lambda$, if $\operatorname{Re}(\lambda) \neq 0$, both the positive and negative real parts are allowed and then the equilibrium configuration is unstable. In fact, the roots of Eq. (30) are

$$
\lambda_{0}=0, \lambda_{1 \pm}= \pm i, \lambda_{2 \pm}= \pm \sqrt{-\frac{1+\sqrt{1-27 V}}{2}}, \lambda_{3 \pm}= \pm \sqrt{-\frac{1-\sqrt{1-27 V}}{2}} .
$$

Therefore, Lagrange's equilateral triangular solution is stable if and only if $\lambda_{2 \pm}$ and $\lambda_{3 \pm}$ are purely imaginary. Hence, it is necessary and sufficient that

$$
1-27 V>0
$$


This is nothing but the Newtonian condition Eq. (1) for stability of Lagrange's solution.

Next, let us consider at the 1PN order. The EIH equations of motion (25) - (28) for the perturbations are

$$
\left(\begin{array}{llll}
M_{11} & M_{12} & M_{13} & M_{14} \\
M_{21} & M_{22} & M_{23} & M_{24} \\
M_{31} & M_{32} & M_{33} & M_{34} \\
M_{41} & M_{42} & M_{43} & M_{44}
\end{array}\right)\left(\begin{array}{c}
\chi_{12} \\
\sigma \\
X \\
\psi_{23}
\end{array}\right)=0
$$

where

$$
\begin{aligned}
M_{11}= & \left(D^{2}-3\right)-\frac{1}{32} \varepsilon\left\{4 \sqrt{3}\left(\nu_{1}-\nu_{2}\right)\left(7-9 \nu_{3}\right) \nu_{3} D+\left(36 \nu_{2}^{3}+234 \nu_{1} \nu_{2}^{2}-146 \nu_{2}^{2}\right.\right. \\
& \left.\left.+261 \nu_{1}^{2} \nu_{2}-488 \nu_{1} \nu_{2}+155 \nu_{2}+63 \nu_{1}^{3}-155 \nu_{1}^{2}+137 \nu_{1}-585\right)\right\}, \\
M_{12}= & -2 D-\frac{1}{24} \varepsilon\left(27 \nu_{2}^{3}+135 \nu_{1} \nu_{2}^{2}-21 \nu_{2}^{2}+135 \nu_{1}^{2} \nu_{2}-210 \nu_{1} \nu_{2}+24 \nu_{2}+27 \nu_{1}^{3}\right. \\
& \left.-21 \nu_{1}^{2}+24 \nu_{1}-155\right) D \\
M_{13}= & -\frac{9}{4} \nu_{3}-\frac{1}{32} \varepsilon \nu_{3}\left\{4 \sqrt{3}\left(9 \nu_{1} \nu_{2}+10 \nu_{2}+9 \nu_{1}^{2}-6 \nu_{1}-4\right) D-\left(216 \nu_{2}^{2}+288 \nu_{1} \nu_{2}\right.\right. \\
& \left.\left.-154 \nu_{2}+171 \nu_{1}^{2}-38 \nu_{1}+420\right)\right\}, \\
M_{14}= & -\frac{3 \sqrt{3}}{4} \nu_{3}+\frac{1}{32} \varepsilon \nu_{3}\left\{4\left(18 \nu_{2}^{2}+27 \nu_{1} \nu_{2}-2 \nu_{2}+9 \nu_{1}^{2}+14 \nu_{1}-12\right) D+\sqrt{3}\left(51 \nu_{2}^{2}\right.\right. \\
& \left.\left.+114 \nu_{1} \nu_{2}+2 \nu_{2}+87 \nu_{1}^{2}-120 \nu_{1}+155\right)\right\}, \\
M_{21}= & 2 D-\frac{1}{32} \varepsilon\left\{4 \left(9 \nu_{2}^{3}+45 \nu_{1} \nu_{2}^{2}+9 \nu_{2}^{2}+45 \nu_{1}^{2} \nu_{2}-30 \nu_{1} \nu_{2}-18 \nu_{2}+9 \nu_{1}^{3}+9 \nu_{1}^{2}\right.\right. \\
& \left.\left.-18 \nu_{1}+61\right) D+3 \sqrt{3} \nu_{3}\left(12 \nu_{2}^{2}-6 \nu_{1} \nu_{2}+14 \nu_{2}-15 \nu_{1}^{2}+4 \nu_{1}-5\right)\right\} \\
& \left.\left.+72 \nu_{1} \nu_{2}-54 \nu_{2}+81 \nu_{1}^{2}-90 \nu_{1}+160\right)\right\}, \\
M_{22}= & D^{2}-\frac{1}{24} \varepsilon\left\{\left(3 \nu_{2}^{2}+12 \nu_{1} \nu_{2}-18 \nu_{2}+3 \nu_{1}^{2}-18 \nu_{1}+10\right) D^{2}-3 \sqrt{3}\left(\nu_{1}-\nu_{2}\right) \nu_{3}\right. \\
& \left.\times\left(9 \nu_{2}+9 \nu_{1}+4\right) D\right\}, \\
M_{23}= & -\frac{3 \sqrt{3}}{4} \nu_{3}+\frac{1}{32} \varepsilon \nu_{3}\left\{4\left(18 \nu_{2}^{2}+27 \nu_{1} \nu_{2}+8 \nu_{2}+9 \nu_{1}^{2}+16 \nu_{1}-12\right) D+\sqrt{3}\left(36 \nu_{2}^{2}\right.\right. \\
& \\
&
\end{aligned}
$$




$$
\begin{aligned}
& M_{24}=\frac{9}{4} \nu_{3}+\frac{1}{32} \varepsilon \nu_{3}\left\{4 \sqrt{3}\left(9 \nu_{1} \nu_{2}+8 \nu_{2}+9 \nu_{1}^{2}-4\right) D-9\left(21 \nu_{2}^{2}+14 \nu_{1} \nu_{2}-10 \nu_{2}\right.\right. \\
& \left.\left.+13 \nu_{1}^{2}-8 \nu_{1}+45\right)\right\} \text {, } \\
& M_{31}=\left(D^{2}-3\right)-\frac{1}{32} \varepsilon\left\{4 \sqrt{3}\left(\nu_{3}-\nu_{1}\right)\left(7-9 \nu_{2}\right) \nu_{2} D+\left(36 \nu_{3}^{3}+234 \nu_{1} \nu_{3}^{2}-146 \nu_{3}^{2}\right.\right. \\
& \left.\left.+261 \nu_{1}^{2} \nu_{3}-488 \nu_{1} \nu_{3}+155 \nu_{3}+63 \nu_{1}^{3}-155 \nu_{1}^{2}+137 \nu_{1}-585\right)\right\}, \\
& M_{32}=-2 D-\frac{1}{24} \varepsilon\left(27 \nu_{3}^{3}+135 \nu_{1} \nu_{3}^{2}-21 \nu_{3}^{2}+135 \nu_{1}^{2} \nu_{3}-210 \nu_{1} \nu_{3}+24 \nu_{3}+27 \nu_{1}^{3}\right. \\
& \left.-21 \nu_{1}^{2}+24 \nu_{1}-155\right) D \\
& M_{33}=D^{2}-3+\frac{9}{4} \nu_{2}-\frac{1}{32} \varepsilon\left\{4 \sqrt{3} \nu_{2}\left(9 \nu_{3}^{2}+9 \nu_{1} \nu_{3}+8 \nu_{3}-4 \nu_{1}-4\right) D-\left(180 \nu_{3}^{3}+270 \nu_{1} \nu_{3}^{2}\right.\right. \\
& \left.\left.-224 \nu_{3}^{2}+198 \nu_{1}^{2} \nu_{3}+8 \nu_{1} \nu_{3}+419 \nu_{3}+108 \nu_{1}^{3}-54 \nu_{1}^{2}+321 \nu_{1}+165\right)\right\}, \\
& M_{34}=-\left(2 D+\frac{3 \sqrt{3}}{4} \nu_{2}\right)+\frac{1}{96} \varepsilon\left\{4 \left(27 \nu_{3}^{3}-39 \nu_{3}^{2}-27 \nu_{1}^{2} \nu_{3}+165 \nu_{1} \nu_{3}-54 \nu_{3}+36 \nu_{1}^{2}\right.\right. \\
& \left.\left.-102 \nu_{1}+191\right) D+3 \sqrt{3} \nu_{2}\left(51 \nu_{3}^{2}+114 \nu_{1} \nu_{3}+2 \nu_{3}+87 \nu_{1}^{2}-120 \nu_{1}+155\right)\right\} \text {, } \\
& M_{41}=2 D-\frac{1}{32} \varepsilon\left\{4 \left(9 \nu_{3}^{3}+45 \nu_{1} \nu_{3}^{2}+9 \nu_{3}^{2}+45 \nu_{1}^{2} \nu_{3}-30 \nu_{1} \nu_{3}-18 \nu_{3}+9 \nu_{1}^{3}+9 \nu_{1}^{2}\right.\right. \\
& \left.\left.-18 \nu_{1}+61\right) D-3 \sqrt{3} \nu_{2}\left(12 \nu_{3}^{2}-6 \nu_{1} \nu_{3}+14 \nu_{3}-15 \nu_{1}^{2}+4 \nu_{1}-5\right)\right\} \text {, } \\
& M_{42}=D^{2}-\frac{1}{24} \varepsilon\left\{\left(3 \nu_{3}^{2}+12 \nu_{1} \nu_{3}-18 \nu_{3}+3 \nu_{1}^{2}-18 \nu_{1}+10\right) D^{2}-3 \sqrt{3}\left(\nu_{3}-\nu_{1}\right)\right. \\
& \left.\times\left(13-9 \nu_{2}\right) \nu_{2} D\right\} \\
& M_{43}=2 D-\frac{3 \sqrt{3}}{4} \nu_{2}+\frac{1}{32} \varepsilon\left\{4\left(9 \nu_{3}^{3}-19 \nu_{3}^{2}-9 \nu_{1}^{2} \nu_{3}+27 \nu_{1} \nu_{3}-2 \nu_{3}-2 \nu_{1}^{2}-10 \nu_{1}-49\right) D\right. \\
& \left.+\sqrt{3}\left(72 \nu_{3}^{2}+54 \nu_{1} \nu_{3}-12 \nu_{3}+36 \nu_{1}^{2}-78 \nu_{1}+145\right) \nu_{2}\right\} \\
& M_{44}=D^{2}-\frac{9}{4} \nu_{2}-\frac{1}{96} \varepsilon\left\{4\left(3 \nu_{3}^{2}+12 \nu_{1} \nu_{3}-18 \nu_{3}+3 \nu_{1}^{2}-18 \nu_{1}+10\right) D^{2}-12 \sqrt{3}\left(9 \nu_{3}^{2}+9 \nu_{1} \nu_{3}\right.\right. \\
& \left.\left.+12 \nu_{3}-4 \nu_{1}-4\right) \nu_{2} D-27\left(21 \nu_{3}^{2}+14 \nu_{1} \nu_{3}-10 \nu_{3}+13 \nu_{1}^{2}-8 \nu_{1}+45\right) \nu_{2}\right\} \text {. }
\end{aligned}
$$

In a similar manner to the Newtonian case, we obtain the secular equation at the $1 \mathrm{PN}$ 
order as

$$
\begin{array}{r}
\lambda^{2}\left[\lambda^{6}+2\left\{1-\frac{1}{8} \varepsilon(77-10 V)\right\} \lambda^{4}+\left\{1+\frac{27}{4} V-\frac{1}{16} \varepsilon\left(308+1265 V+162 W-378 V^{2}\right)\right\} \lambda^{2}\right. \\
\left.+\frac{27}{4}\left\{V-\frac{1}{24} \varepsilon\left(521 V-72 W-126 V^{2}\right)\right\}\right]=0
\end{array}
$$

where $W \equiv \nu_{1} \nu_{2} \nu_{3}$.

Neglecting the trivial root $\lambda=0$, we obtain a cubic equation of $\tau \equiv \lambda^{2}$ as

$$
\tau^{3}+\alpha \tau^{2}+\beta \tau+\gamma=0,
$$

where

$$
\begin{aligned}
& \alpha \equiv 2\left\{1-\frac{1}{8} \varepsilon(77-10 V)\right\}, \\
& \beta \equiv 1+\frac{27}{4} V-\frac{1}{16} \varepsilon\left(308+1265 V+162 W-378 V^{2}\right), \\
& \gamma \equiv \frac{27}{4}\left\{V-\frac{1}{24} \varepsilon\left(521 V-72 W-126 V^{2}\right)\right\} .
\end{aligned}
$$

In a similar manner to the Newtonian case, the PN triangular solution is stable if and only if all the roots $\tau$ of Eq. (51) are negative real, so that all $\lambda= \pm \sqrt{\tau}$ have no real part.

In the 1PN approximation, the PN corrections to the Newtonian roots must be small. Thus, we can factor Eq. (51) as

$$
(\tau+1-a \varepsilon)\left(\tau^{2}+b \tau+c\right)=0,
$$

where $a, b$, and $c$ are constants and the 2PN terms are neglected. From Eqs. (51) and (55), one can obtain

$$
\begin{aligned}
& a=\frac{1}{8 V}\left(77 V-14 V^{2}-36 W\right), \\
& b=1-\frac{1}{8 V}\left(77 V-6 V^{2}+36 W\right) \varepsilon, \\
& c=\frac{27}{4} V-\frac{1}{16}\left(1305 V-378 V^{2}+162 W\right) \varepsilon .
\end{aligned}
$$

Since $\varepsilon \ll 1$, we have $-1+a \varepsilon<0, b>0$, and $c>0$. The roots of Eq. (55) are expressed as

$$
\tau_{1}=-1+a \varepsilon, \tau_{ \pm}=\frac{-b \pm \sqrt{b^{2}-4 c}}{2} .
$$


Hence, Eq. (55) has three negative real roots if and only if all the roots (59) are negative real. Namely, it is necessary and sufficient for the stability that

$$
b^{2}-4 c>0
$$

For the critical value $b^{2}-4 c=0, V$ in the PN terms of Eq. (60) with Eqs. (57) and (58) can be replaced by the Newtonian critical value as $V=1 / 27$, because $1 \mathrm{PN}$ corrections to it make $2 \mathrm{PN}$ (or higher-order) contributions and they can be neglected. Therefore, the condition for stability of the PN triangular solution becomes

$$
1-\frac{391}{54} \varepsilon-27\left(V+\frac{15}{2} W \varepsilon\right)>0 .
$$

This is explicitly rewritten as

$$
\frac{m_{1} m_{2}+m_{2} m_{3}+m_{3} m_{1}}{\left(m_{1}+m_{2}+m_{3}\right)^{2}}+\frac{15}{2} \frac{m_{1} m_{2} m_{3}}{\left(m_{1}+m_{2}+m_{3}\right)^{3}} \varepsilon<\frac{1}{27}\left(1-\frac{391}{54} \varepsilon\right) .
$$

Equation (62) recovers the Newtonian condition (11) in the limit $\varepsilon \rightarrow 0$. The PN correction in the right-hand side of Eq. (62) is negative and the PN term of the triple product of masses in the left-hand side of Eq. (62) is positive. Hence, the PN condition for stability is tighter than the Newtonian one for any positive small value of the parameter $\varepsilon$. Figure 3 shows the Newtonian stability regions Eq. (1) and the $1 \mathrm{PN}$ ones Eq. (62) when $\varepsilon=0.01$ (i.e. the order of magnitude of the PN effects is 0.01), for instance.

Finally, we focus on the restricted three-body limit as $\nu_{3} \rightarrow 0$ (i.e. $W \rightarrow 0$ ). In this case, the stability condition Eq. (60) becomes

$$
1-27 V-\frac{77-1311 V+378 V^{2}}{4} \varepsilon>0
$$

This is a quadratic inequality of $V$. Solving Eq. (63) for $V$, we obtain

$$
\frac{m_{1} m_{2}}{\left(m_{1}+m_{2}\right)^{2}}<\frac{1}{27}\left(1-\frac{391}{54} \varepsilon\right)
$$

where we used a relation $0 \leq V \leq 1 / 4$ in the restricted three-body problem. Using a relation $\nu_{1}+\nu_{2}=1$ and assuming $\nu_{1}>\nu_{2}$ without loss of generality, we can rewrite Eq. (64) as

$$
\frac{m_{2}}{m_{1}+m_{2}}<\mu_{0}-\frac{17 \sqrt{69}}{486} \varepsilon
$$

where the Newtonian value $\mu_{0}=(9-\sqrt{69}) / 18$. This condition is in agreement with previous results [24-26] (see Eq. (3) in the present paper). Figure 4 shows a region of stability in the $\mathrm{PN}$ restricted three-body problem. 


\section{CONCLUSION}

We examined the PN effects on the stability of the triangular solution in the relativistic three-body problem for general masses. The stability regions still exist even at the $1 P N$ order. The PN stability condition Eq. (62) is consistent with the Newtonian one Eq. (11) in the limit as $\varepsilon \rightarrow 0$. The PN correction in the right-hand side of Eq. (62), which is in agreement with that in the PN restricted three-body problem [24 26], makes the condition more strict than the Newtonian case for any small positive value of the parameter $\varepsilon$.

The PN term of the triple product of masses in the left-hand side of Eq. (62) appears not in the restricted case but in the general one. The instability is also enhanced by this term, while this effect is smaller than the other PN one in the case of mass ratios for stable configurations. If a system is mildly relativistic as $\varepsilon=0.01$, for instance, the maximum value of $W$ is $\mathrm{O}\left(10^{-4}\right)$ when $\nu_{2}=\nu_{3} \approx 0.019$ in a stability region. Namely, the contribution from $W$ is comparable to the $2 \mathrm{PN}$ (or more higher) order. This implies that triple systems with the PN triangular configuration for three finite masses are possible as well as restricted three-body systems.

The PN triangular configuration ought to emit gravitational waves [7, 10]. Such a system will shrink by gravitational radiation reaction if its configuration is initially stable, and the PN effects on the long-term stability should be incorporated. In addition, we concentrate on the circular orbit in this paper. Gravitational radiation is known to decrease eccentricity in binary orbits. It is left as a future work to study gravitational radiation reaction to the PN triangular configuration.

\section{Acknowledgments}

We would like to thank Taihei Yano and Haruo Yoshida for providing with useful information on the literature. We are grateful to Yuuiti Sendouda for useful comments. This work was supported in part by JSPS Grant-in-Aid for JSPS Fellows, No. 24108 (K.Y.), and JSPS Grant-in-Aid for Scientific Research (Kiban C), No. 26400262 (H.A.). 


\section{Appendix A: A Derivation of the Equations of Motion for Perturbations}

We consider four perturbations in the orbital plane (see Fig. 2). First, we put the distances between the bodies as

$$
r_{I J}=\ell\left(1+\rho_{I J}+\delta \chi_{I J}\right)
$$

where $\chi_{I J}\left(=\chi_{J I}\right)$ is a perturbation in the distance $r_{I J}$ and $\delta$ is a bookkeeping parameter that denotes the smallness of the perturbations. By these perturbations, each angle $\varphi_{I J}$ between $\boldsymbol{r}_{K I}$ and $\boldsymbol{r}_{J K}(I \neq J \neq K)$ of the PN triangle is changed as

$$
\varphi_{I J}=\frac{\pi}{3}+\sqrt{3} \rho_{I J}+\delta \psi_{I J}
$$

The perturbations $\chi_{I J}$ and $\psi_{I J}$ relate to each other through the cosine formula, and the number of independent perturbations is three.

The remaining one of the degrees of freedom corresponds to a change in the angular velocity of the bodies:

$$
\theta_{I J}=\Theta_{I J}+\delta \sigma_{I J}
$$

where $\theta_{I J}$ and $\sigma_{I J}$ denote the direction of $\boldsymbol{r}_{I J}$ to the reference frame and a perturbation in it, respectively. $\Theta_{I J}$ is the unperturbed direction which satisfies the equation as

$$
\frac{d \Theta_{I J}}{d t}=\omega_{\mathrm{N}}\left(1+\tilde{\omega}_{\mathrm{PN}}\right)
$$

Differentiating relations as

$$
\begin{aligned}
& \theta_{23}=\theta_{12}-\pi-\varphi_{31} \\
& \theta_{31}=\theta_{12}+\pi+\varphi_{23}
\end{aligned}
$$

we obtain

$$
\begin{aligned}
& D \sigma_{23}=D\left(\sigma-\psi_{31}\right), \\
& D \sigma_{31}=D\left(\sigma+\psi_{23}\right),
\end{aligned}
$$

where $D$ denotes a differential operator with respect to a normalized time $\tilde{t} \equiv \omega_{\mathrm{N}} t$ and we denote $\sigma_{12}$ simply as $\sigma$. Thus, the number of degrees of freedom for $\left(\sigma_{12}, \sigma_{23}, \sigma_{31}\right)$ is one and it corresponds to a change in the angular velocity. 
Note that the perturbations have not only the Newtonian terms but also the 1PN ones. For instance, the perturbation $\sigma$ can be expanded as

$$
\sigma=\sigma_{\mathrm{N}}+\sigma_{\mathrm{PN}}
$$

where $\sigma_{\mathrm{N}}$ and $\sigma_{\mathrm{PN}}(=\mathrm{O}(\varepsilon))$ are the Newtonian term and the $1 \mathrm{PN}$ one, respectively. In the following, we neglect the terms of second (and higher) order in $\delta$. Namely, we calculate to the terms of order $\varepsilon \times \delta$ (i.e. the linear perturbation at the $1 \mathrm{PN}$ order).

Using a complex plane as the orbital one, we denote the relative position of the bodies as $\boldsymbol{r}_{I J} \rightarrow z_{I J}=r_{I J} e^{i \theta_{I J}}$. The EIH equation of motion for $z_{12}$ becomes

$$
\frac{d^{2} z_{12}}{d t^{2}}=F_{12} e^{i \theta_{12}}
$$

The left-hand side of this equation is

$$
\begin{aligned}
\frac{d^{2} z_{12}}{d t^{2}}= & \ell \omega_{\mathrm{N}}^{2}\left[-\left\{1+2 \tilde{\omega}_{\mathrm{PN}}+\rho_{12}+\delta\left(2 D \sigma_{12}+\chi_{12}-D^{2} \chi_{12}+2 \tilde{\omega}_{\mathrm{PN}} D \sigma_{12}+2 \rho_{12} D \sigma_{12}\right.\right.\right. \\
& \left.\left.\left.+2 \tilde{\omega}_{\mathrm{PN}} \chi_{12}\right)\right\}+i \delta\left(2 D \chi_{12}+D^{2} \sigma_{12}+2 \tilde{\omega}_{\mathrm{PN}} D \chi_{12}+\rho_{12} D^{2} \sigma_{12}\right)\right] e^{i \theta_{12}} .
\end{aligned}
$$

$F_{12}$ in the right-hand side of the equation of motion can be expanded as

$$
F_{12}=F_{\mathrm{N} 12}+\varepsilon F_{\mathrm{PN} 12}+\delta F_{\mathrm{Nper} 12}+\varepsilon \delta F_{\mathrm{PNper} 12}
$$

where $F_{\mathrm{N} 12}$ and $F_{\mathrm{PN} 12}$ are the unperturbed Newtonian and PN terms, respectively, and $F_{\text {Nper 12 }}$ and $F_{\text {PNper 12 }}$ are the perturbed Newtonian and PN terms, respectively. These are

$$
\begin{aligned}
F_{\mathrm{N} 12} & =-\frac{M}{\ell^{2}}, \\
F_{\mathrm{PN} 12} & =\frac{1}{24} \frac{M}{\ell^{2}}\left(45 \nu_{2}^{2}+54 \nu_{1} \nu_{2}-60 \nu_{2}+45 \nu_{1}^{2}-60 \nu_{1}+97\right), \\
F_{\mathrm{Nper} 12} & =\frac{1}{2} \frac{M}{\ell^{2}}\left[3 \nu_{3}\left(\chi_{23}+\chi_{31}\right)+2\left(2-3 \nu_{3}\right) \chi_{12}\right]+i \frac{3 \sqrt{3}}{2} \frac{M}{\ell^{2}} \nu_{3}\left(\chi_{31}-\chi_{23}\right),
\end{aligned}
$$




$$
\begin{aligned}
F_{\mathrm{PNper} 12}= & \frac{1}{16} \frac{M}{\ell^{2}}\left[-2\left(54 \nu_{2}^{3}+108 \nu_{1} \nu_{2}^{2}-86 \nu_{2}^{2}+108 \nu_{1}^{2} \nu_{2}-82 \nu_{1} \nu_{2}+167 \nu_{2}+54 \nu_{1}^{3}\right.\right. \\
& \left.-86 \nu_{1}^{2}+167 \nu_{1}-29\right) \chi_{12}-\nu_{3}\left(45 \nu_{2}^{2}+108 \nu_{1} \nu_{2}+8 \nu_{2}+90 \nu_{1}^{2}-108 \nu_{1}+150\right) \\
& \times \chi_{23}-\nu_{3}\left(90 \nu_{2}^{2}+108 \nu_{1} \nu_{2}-108 \nu_{2}+45 \nu_{1}^{2}+8 \nu_{1}+150\right) \chi_{31}+8\left(\nu_{2}^{3}-\nu_{1} \nu_{2}^{2}\right. \\
& \left.+2 \nu_{2}^{2}-\nu_{1}^{2} \nu_{2}-4 \nu_{1} \nu_{2}-7 \nu_{2}+\nu_{1}^{3}+2 \nu_{1}^{2}-7 \nu_{1}\right) D \sigma_{12}-2 \nu_{3}\left(\nu_{2}^{2}+22 \nu_{1} \nu_{2}+4 \nu_{2}\right. \\
& \left.+4 \nu_{1}^{2}+2 \nu_{1}+8\right) D \sigma_{23}-2 \nu_{3}\left(4 \nu_{2}^{2}+22 \nu_{1} \nu_{2}+2 \nu_{2}+\nu_{1}^{2}+4 \nu_{1}+8\right) D \sigma_{31} \\
& +8 \sqrt{3} \nu_{3}\left(\nu_{1}-\nu_{2}\right)\left(3-\nu_{3}\right) D \chi_{12}-2 \sqrt{3} \nu_{3}\left(9 \nu_{2}^{2}-4 \nu_{1} \nu_{2}-4 \nu_{2}+4 \nu_{1}^{2}+6 \nu_{1}\right. \\
& \left.-16) D \chi_{23}+2 \sqrt{3} \nu_{3}\left(4 \nu_{2}^{2}-4 \nu_{1} \nu_{2}+6 \nu_{2}+9 \nu_{1}^{2}-4 \nu_{1}-16\right) D \chi_{31}\right] \\
& +i \frac{1}{16} \frac{M}{\ell^{2}}\left[-12 \sqrt{3} \nu_{3}\left(\nu_{1}-\nu_{2}\right)\left(3-\nu_{3}\right) \chi_{12}+\sqrt{3} \nu_{3}\left(57 \nu_{2}^{2}+36 \nu_{1} \nu_{2}-24 \nu_{2}\right.\right. \\
& \left.+42 \nu_{1}^{2}-12 \nu_{1}+130\right) \chi_{23}-\sqrt{3} \nu_{3}\left(42 \nu_{2}^{2}+36 \nu_{1} \nu_{2}-12 \nu_{2}+57 \nu_{1}^{2}-24 \nu_{1}\right. \\
& +130) \chi_{31}-8 \sqrt{3} \nu_{3}\left(\nu_{1}-\nu_{2}\right)\left(\nu_{1}+\nu_{2}\right) D \sigma_{12}+2 \sqrt{3} \nu_{3}\left(\nu_{2}^{2}-12 \nu_{1} \nu_{2}+14 \nu_{2}\right. \\
& \left.-4 \nu_{1}^{2}+10 \nu_{1}+8\right) D \sigma_{23}+2 \sqrt{3} \nu_{3}\left(4 \nu_{2}^{2}+12 \nu_{1} \nu_{2}-10 \nu_{2}-\nu_{1}^{2}-14 \nu_{1}-8\right) D \sigma_{31} \\
& -8\left(3 \nu_{2}^{3}+9 \nu_{1} \nu_{2}^{2}-6 \nu_{2}^{2}+9 \nu_{1}^{2} \nu_{2}-8 \nu_{1} \nu_{2}-5 \nu_{2}+3 \nu_{1}^{3}-6 \nu_{1}^{2}-5 \nu_{1}\right) D \chi_{12} \\
& -2 \nu_{3}\left(9 \nu_{2}^{2}+30 \nu_{1} \nu_{2}+10 \nu_{2}+12 \nu_{1}^{2}-18 \nu_{1}-16\right) D \chi_{23}-2 \nu_{3}\left(12 \nu_{2}^{2}+30 \nu_{1} \nu_{2}\right. \\
& \left.\left.-18 \nu_{2}+9 \nu_{1}^{2}+10 \nu_{1}-16\right) D \chi_{31}\right] .
\end{aligned}
$$

For exchanging indices between 1 and 2 such as $\nu_{1} \leftrightarrow \nu_{2}$ and $\chi_{31} \leftrightarrow \chi_{23}$, we have the symmetry/antisymmetry in the real/imaginary parts of Eqs. (A13) - (A16). This (anti)symmetry may ensure the form of the equations, though they are rather complicated. We can obtain the EIH equations of motion for $z_{23}$ and $z_{31}$ by the cyclic manipulations as $1 \rightarrow 2 \rightarrow 3 \rightarrow 1$. Since the unperturbed terms in the equations give the PN triangular equilibrium solution, we focus on the perturbed terms. It is convenient to transform the variables as [23]

$$
\begin{aligned}
\chi_{23} & =\frac{1}{2}\left[\left(1-3 \rho_{12}\right) \chi_{31}+\left(1-3 \rho_{31}\right) \chi_{12}+\sqrt{3}\left(1-\rho_{23}\right) \psi_{23}\right], \\
X & \equiv \chi_{31}-\chi_{12} .
\end{aligned}
$$

Using the relations Eqs. (A7), (A8), (A17), and (A18), we obtain the equations of motion 
(25) - (28) for perturbations.

[1] H. Goldstein, Classical Mechanics (Addison-Wesley, MA, 1980).

[2] J. M. A. Danby, Fundamentals of Celestial Mechanics (William-Bell, VA, 1988).

[3] C. Marchal, The Three-Body Problem (Elsevier, Amsterdam, 1990).

[4] M. Connors, P. Wiegert and C. Veillet, Nature 475, 481 (2011).

[5] E. Krefetz, Astron. J 72, 471 (1967).

[6] T. I. Maindl, Completing the Inventory of the Solar System, Astronomical Society of the Pacific Conference Proceedings, edited by T.W. Rettig and J.M. Hahn, 107, 147 (1996).

[7] H. Asada, Phys. Rev. D 80064021 (2009).

[8] N. Seto and T. Muto, Phys. Rev. D 81103004 (2010).

[9] J. D. Schnittman, Astrophys. J. 72439 (2010).

[10] Y. Torigoe, K. Hattori, and H. Asada, Phys. Rev. Lett. 102, 251101 (2009).

[11] K. Nordtvedt, Phys. Rev. 1691014 (1968).

[12] O. Blaes, M. H. Lee, and A. Socrates, Astrophys. J. 578, 775 (2002).

[13] M. C. Miller and D. P. Hamilton, Astrophys. J. 576, 894 (2002).

[14] L. Wen, Astrophys. J. 598, 419 (2003)

[15] T. A. Thompson, Astrophys. J. 741, 82 (2011).

[16] N. Seto, Phys. Rev. Lett. 111, 061106 (2013).

[17] S. M. Ransom et al., Nature 505, 520 (2014).

[18] K. Yamada and H. Asada, Phys. Rev. D 82, 104019 (2010).

[19] K. Yamada and H. Asada, Phys. Rev. D 83, 024040 (2011).

[20] T. Ichita, K. Yamada, and H. Asada, Phys. Rev. D 83, 084026 (2011).

[21] K. Yamada and H. Asada, Phys. Rev. D 86, 124029 (2012).

[22] G. Gascheau, C. R. Acad. Sci. 16, 393 (1843).

[23] R. J. Routh, Proc. Lond. Math. Soc. 6, 86 (1875).

[24] C. N. Douskos and E.A. Perdios, Celest. Mech. Dyn. Astron. 82, 317 (2002).

[25] J. Singh and N. Bello, Astrophys. Space Sci., 351, 483, (2014).

[26] J. Singh and N. Bello, Astrophys. Space Sci., 351, 491, (2014).

[27] C. W. Misner, K. S. Thorne, and J. A. Wheeler, Gravitation, (Freeman, New York, 1973). 
[28] L. D. Landau and E. M. Lifshitz, The Classical Theory of Fields (Oxford, Pergamon 1962).

[29] C. M. Will, Theory and experiment in gravitational physics (Cambridge University, New York, 1993).

[30] H. Asada, T. Futamase, and P. Hogan, Equations of Motion in General Relativity (Oxford University, New York, 2011). 


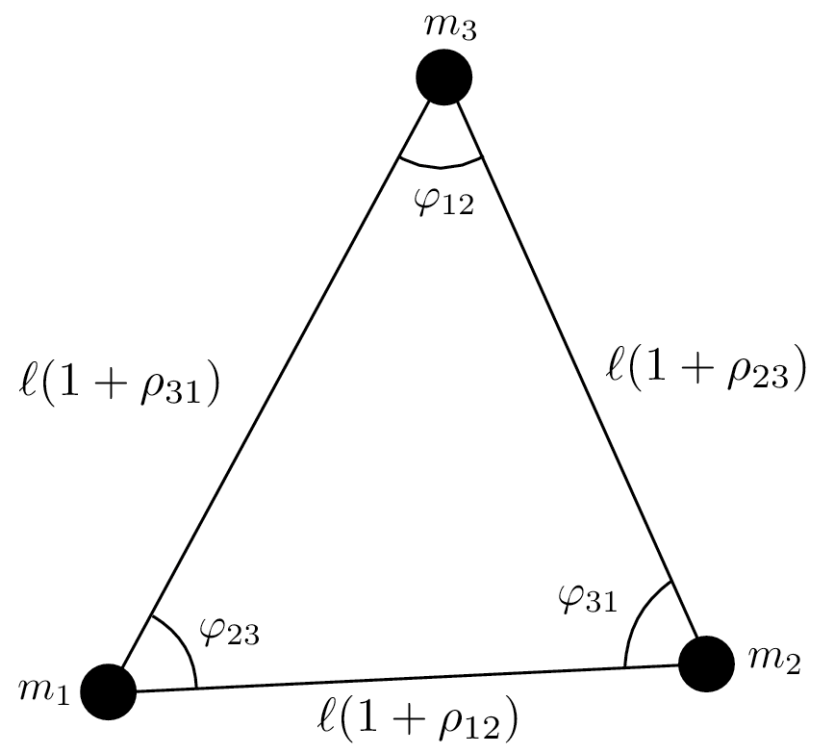

FIG. 1: PN triangular configuration. Each body is located at one of the apexes. $\rho_{I J}$ denotes the $\mathrm{PN}$ corrections to each side length at the $1 \mathrm{PN}$ order. In the equilateral case, $\rho_{12}=\rho_{23}=\rho_{31}=0$, namely, $r_{12}=r_{23}=r_{31}=\ell$ according to Eq. (10). 


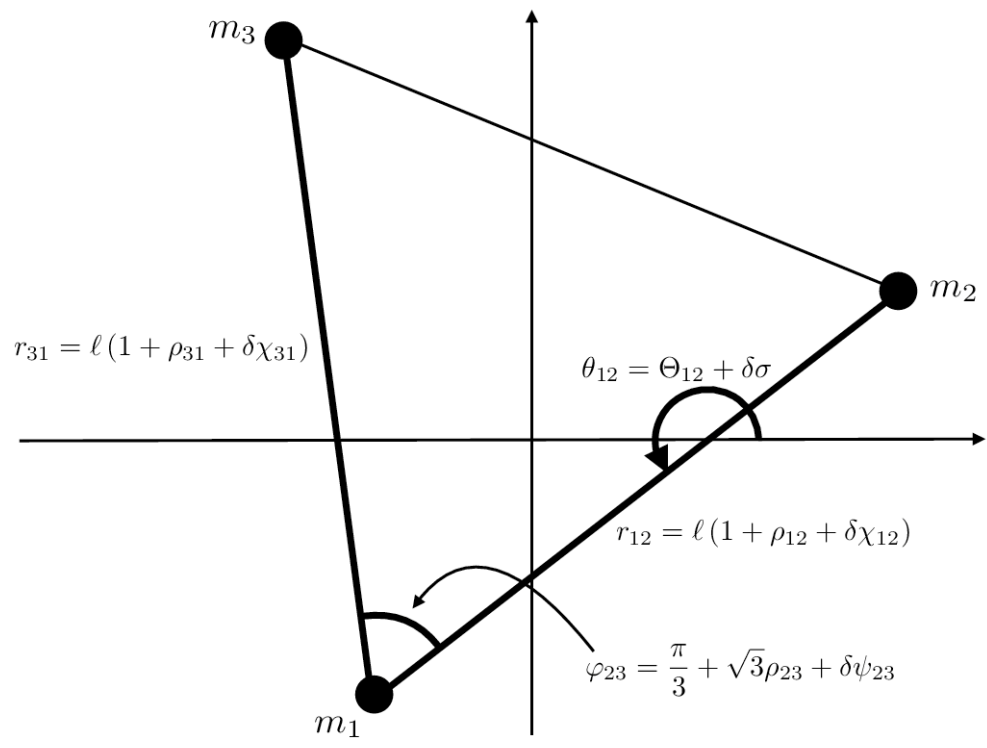

FIG. 2: Four perturbations in the PN triangular configuration. The perturbations $\chi_{12}$ and $\chi_{31}$ denote changes in $r_{12}$ and $r_{31}$, respectively, $\psi_{23}$ is a perturbation in the interior angle $\varphi_{23}$, and $\sigma$ corresponds to a change in the angular velocity. 

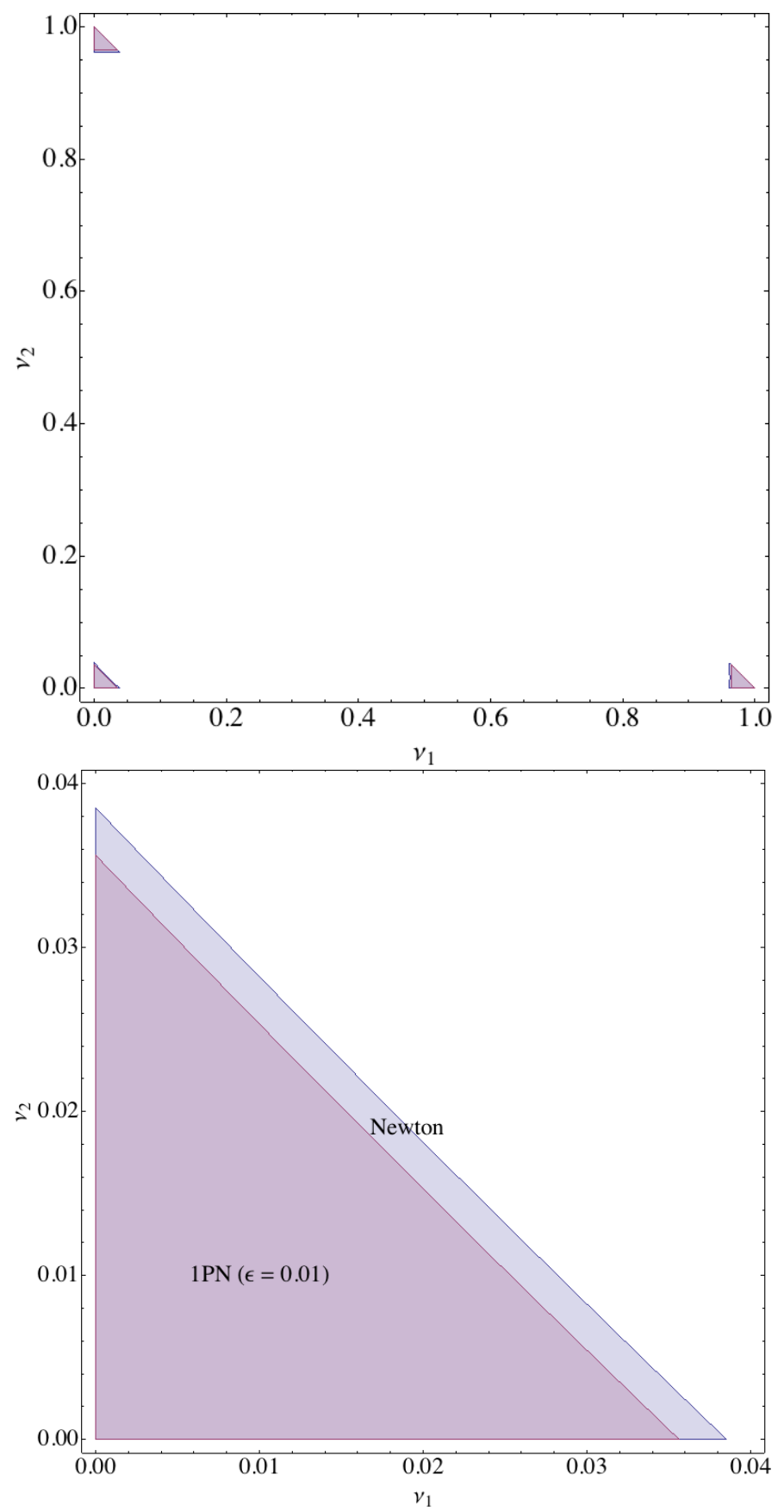

FIG. 3: Mass ratios for a stable configuration that satisfies the Newtonian condition Eq. (1) and the PN one Eq. (62) when $\varepsilon=0.01$ (i.e. the order of magnitude of the $1 \mathrm{PN}$ effects is 0.01 ), for instance. For values of the mass ratios within the colored areas, the triangular configuration for three finite masses is stable. Top: All the stability regions. Bottom: The regions around small $\nu_{1}$ and $\nu_{2}$, where the third mass is dominant. The stability regions at the $1 \mathrm{PN}$ order still exist, though they are more narrow than the Newtonian case. 


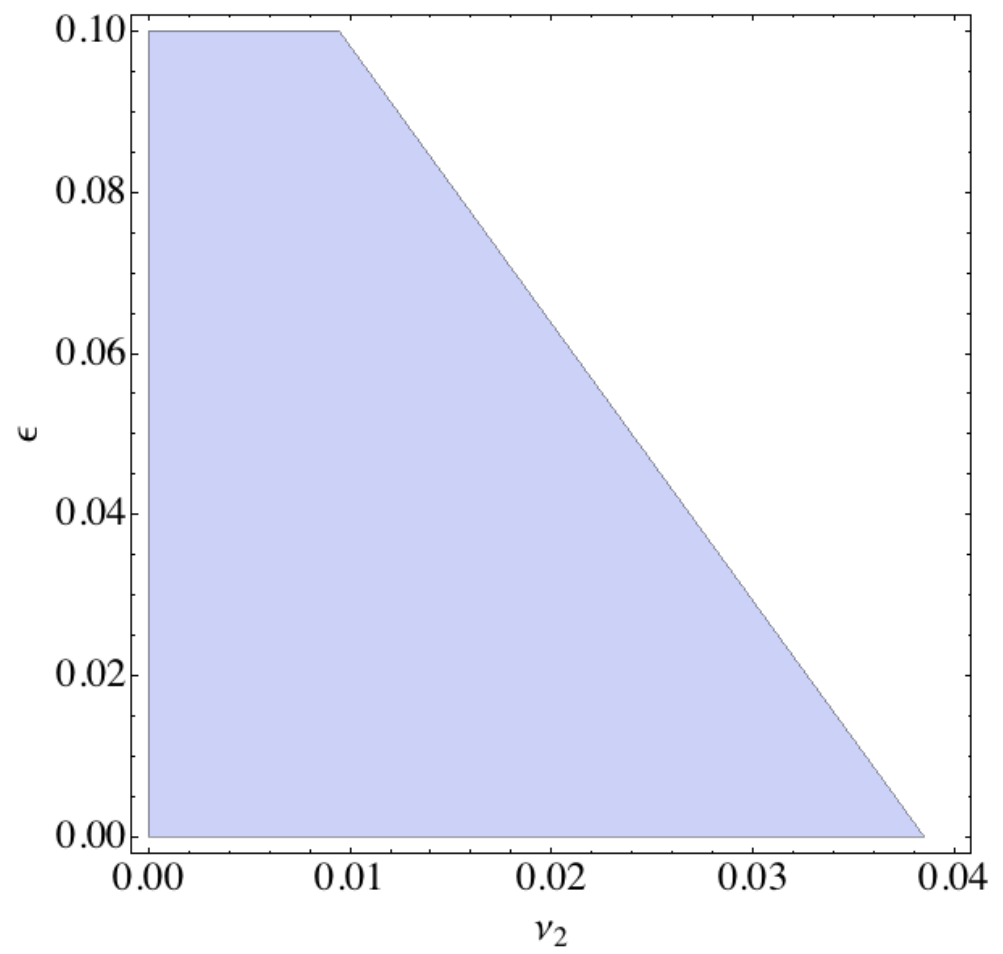

FIG. 4: The stability region Eq. (65) for the PN restricted three-body systems in the $\left(\nu_{2}, \varepsilon\right)$ plane. For values of $\nu_{2}$ and $\varepsilon$ within the colored area, the $\mathrm{PN}$ triangular configuration in the restricted three-body case is stable. This figure corresponds to Fig. 1 in Ref. [24]. 